\title{
Chimiothérapie des tumeurs germinales du testicule
}

\author{
Stéphane CULINE
}

CRLC val d'Aurelle, Montpellier

\section{RESUME}

La chimiothérapie de référence des tumeurs germinales du testicule repose sur trois molécules majeures : la bléomycine, l'étoposide et le cisplatine (protocole BEP). Le nombre de cycles recommandé est variable selon la situation clinique : deux pour les tumeurs non séminomateuses de stades localisés à haut risque d'évolutivité métastatique, trois pour les séminomes de stades avancés et les tumeurs non séminomateuses métastatiques de bon pronostic, quatre pour les tumeurs non séminomateuses de pronostic intermédiaire et de mauvais pronostic. La chirurgie d'exérèse des lésions métastatiques résiduelles doit être envisagée après la normalisation des marqueurs tumoraux sériques pour les tumeurs non séminomateuses. Les taux de guérison obtenus sont très élevés, de l'ordre de $\mathbf{9 0 \%}$ pour l'ensemble des tumeurs germinales. Ils ne peuvent être atteints que par la connaissance optimale des facteurs pronostiques et le respect impératif des modalités pratiques de délivrance des traitements. Tout écart aux standards établis peut avoir pour conséquences de diminuer l'efficacité ou de majorer les toxicités attendues.

Mots clés : cancer du testicule, tumeur germinale, chimiothérapie

\section{INTRODUCTION}

En 2002, la prise en charge des tumeurs germinales du testicule permet d'obtenir des taux de guérison d'environ 90\%. Depuis l'introduction du cisplatine dans les protocoles de chimiothérapie à la fin des années 1970, vingt ans de recherche clinique ont permis de définir les variables pronostiques et les traitements optimaux qui ont fait de cette pathologie un modèle, encore trop rare en cancérologie, de curabilité, même à des stades avancés de la maladie [1].

\section{LES PROTOCOLES}

Trois drogues apparaissent prédominantes : le cisplatine bien sûr, l'étoposide et la bléomycine. Leur association définit le classique protocole BEP, dont les modalités d'administration sont détaillées dans le tableau 1 et doivent être rigoureusement respectées. Tout écart risque en effet d'en compromettre l'efficacité et donc de réduire les espoirs de guérison. Une hyperhydration salée quotidienne est obligatoire pendant chaque cure afin de prévenir la toxicité rénale du cisplatine, de sorte que l'administration en hôpital de jour n'est pas conseillée. Afin de limiter les toxicités hématologiques, la prescription de facteurs de croissance hématopoïétiques (G-CSF et érythropoiétine) est fréquemment associée. Le protocole BEP représente le standard pour la prise en charge de première intention. En fonction du contexte, le nombre de cycles optimal varie de 2 à 4 .

Chez les patients en échec d'une première ligne de traitement par le protocole BEP, l'association historique de réfé-

\section{Correspondance :}

Pr Stéphane Culine - Département d'Oncologie Médicale, C.R.L.C. Val d'Aurelle, Parc Euromédecine, 34298 - MONTPELLIER Cedex 5 - Tel 0467613152 - Fax 33467613022 Email stculine@valdorel.fnclcc.fr 
rence est le VeIP (Tableau 1). Au cours des dernières années, l'activité de trois molécules cytotoxiques, la gemcitabine, l'oxaliplatine et le paclitaxel, a été démontrée en situation de rattrapage. Des études complémentaires devront définir leur place précise dans la stratégie thérapeutique [2].

Tableau 1 : Protocoles de chimiothérapie des tumeurs germinales.

\begin{tabular}{lll}
\hline & Protocole BEP \\
\hline Bléomycine & $30 \mathrm{mg} / \mathrm{j}$ dose totale & J1, J8, \\
& $\mathrm{J} 15$ \\
Etoposide & $100 \mathrm{mg} / \mathrm{m}^{2} / \mathrm{j}$ & $\mathrm{J} 1$ à $\mathrm{J} 5$ \\
Cisplatine & $20 \mathrm{mg} / \mathrm{m}^{2} / \mathrm{j}$ & $\mathrm{J} 1$ à $\mathrm{J} 5$ \\
Chaque premier jour de cycle est espacé de 21 jours. \\
\hline
\end{tabular}

\section{Protocole VeIP}

$\begin{array}{lll}\text { Vinblastine } & 0,11 \mathrm{mg} / \mathrm{kg} / \mathrm{j} & \mathrm{J} 1 \text { et } \mathrm{J} 2 \\ \text { Ifosfamide } & 1200 \mathrm{mg} / \mathrm{m}^{2} / \mathrm{j} & \mathrm{J} 1 \text { à } 5 \\ \text { Cisplatine } & 20 \mathrm{mg} / \mathrm{m}^{2} / \mathrm{j} & \mathrm{J} 1 \text { à } \mathrm{J} 5\end{array}$

Chaque premier jour de cycle est espacé de 21 jours.

\section{LES INDICATIONS}

La chimiothérapie est actuellement indiquée, après 1'orchidectomie, dans les stades métastatiques des tumeurs germinales du testicule, mais également dans les stades localisés des tumeurs non séminomateuses à haut risque d'évolutivité métastatique [1].

\section{Les tumeurs non séminomateuses métastatiques}

Le nombre de cycles de BEP est dicté par les facteurs pronostiques établis par la classification internationale (Tableau 2) [3]. Trois cycles sont suffisants pour les tumeurs de bon pronostic, alors que quatre cycles sont nécessaires pour les patients de pronostic intermédiaire ou de mauvais pronostic. L'objectif premier de la chimiothérapie est la normalisation des marqueurs tumoraux sériques. L'évaluation tomodensitométrique permet alors de porter les indications d'exérèse chirurgicale des lésions métastatiques résiduelles. Toute masse tumorale supra-centimétrique persistant après la chimiothérapie doit a priori faire l'objet d'une chirurgie d'exérèse.

\section{Les tumeurs non séminomateuses localisées}

Les stades localisés sont définis par l'absence de métastase décelable sur le bilan d'extension tomodensitométrique et la normalisation des marqueurs tumoraux sériques après l'orchidectomie. La chimiothérapie doit être réservée aux formes à haut risque d'évolutivité métastatique, définies par deux caractéristiques précisées par l'expertise anatomo-pathologique faite sur la pièce opératoire : la présence d'embols tumoraux intra-vasculaires et/ou une proportion importante (en pratique, supérieure à $80 \%$ ) de la composante de carcinome embryonnaire au sein de la tumeur. Deux cycles du protocole BEP sont suffisants dans ce contexte.

\section{Les tumeurs séminomateuses pures de stades avancés}

La radiothérapie demeure le traitement de référence des stades précoces des tumeurs séminomateuses pures (stades localisés au testicule ou extension métastatique ganglionnaire lombo-aortique de moins de $5 \mathrm{~cm}$ de diamètre transversal). Les indications de chimiothérapie sont donc les stades avancés de la maladie : métastases ganglionnaires lombo-aortiques de plus de $5 \mathrm{~cm}$ de diamètre transversal ou extension métastatique hépatique ou sus-diaphragmatique. Trois cycles de BEP (ou 4 cycles du même protocole sans bléomycine) constituent le traitement de référence. Les indications chirurgicales d'exérèse des lésions métastatiques résiduelles après la chimiothérapie sont réservées aux masses de plus de $3 \mathrm{~cm}$ de diamètre dont la résorption progressive au cours du temps n'est pas jugée satisfaisante.

\section{Les traitements de rattrapage}

La chimiothérapie de rattrapage est indiquée lorsque la maladie germinale n'a pas été mise en rémission ou récidive après une première ligne de traitement. Quatre cycles du protocole VeIP constituent la référence actuelle, suivis de l'exérèse chirurgicale des lésions résiduelles. Il n'y a pas de place, hors essai thérapeutique, pour la délivrance d'une chimiothérapie à fortes doses avec support hématopoïétique [4].

\section{LES RESULTATS}

Les taux attendus de guérison sont de l'ordre de plus de $90 \%$ pour les tumeurs séminomateuses pures et non séminomateuses de bon pronostic, et d'environ $80 \%$ et $50 \%$ à $60 \%$ pour les patients de pronostic intermédiaire et de mauvais pronostic, respectivement. La chimiothérapie adjuvante des stades localisés des tumeurs non séminomateuses permet de guérir plus de $95 \%$ des patients. Les taux de guérison après les traitements de rattrapage sont de l'ordre de $50 \%$ pour les tumeurs séminomateuses pures, et de $25 \%$ pour les tumeurs non séminomateuses.

\section{CONCLUSION}

L'objectif de la prise en charge des patients présentant une tumeur germinale du testicule est la guérison. Il ne peut être atteint que par la connaissance optimale des facteurs pronostiques et le respect impératif des modalités pratiques de délivrance des traitements. Tout écart aux standards établis peut avoir pour conséquences de diminuer l'efficacité en cas de sous-dosages ou de majorer la toxicité en cas de surdosages. 
Tableau 2 : Classification pronostique internationale des tumeurs germinales non séminomateuses du testicule.

\begin{tabular}{|c|c|c|}
\hline Pronostic & Caractéristiques & Survie à 5 ans \\
\hline BON & $\begin{array}{c}\text { Absence de métastases } \\
\text { viscérales extra-pulmonaires } \\
\text { et } \\
\begin{array}{c}\mathrm{AFP}<1000 \mathrm{ng} / \mathrm{ml} \\
\text { et } \\
\mathrm{HCG}<5000 \mathrm{UI} / \mathrm{l} \\
\text { et } \\
\mathrm{LDH}<1,5 \times \mathrm{N}\end{array}\end{array}$ & $92 \%$ \\
\hline INTERMEDIAIRE & $\begin{array}{c}\text { Absence de métastases } \\
\text { viscérales extra-pulmonaires } \\
\text { et } \\
1000 \leq \mathrm{AFP} \leq 10000 \mathrm{ng} / \mathrm{ml} \\
\text { et } \\
5000 \leq \mathrm{HCG} \leq 50000 \mathrm{UI} / 1 \\
\text { et } \\
1,5 \leq \mathrm{LDH}<10 \times \mathrm{N}\end{array}$ & $80 \%$ \\
\hline MAUVAIS & $\begin{array}{l}\text { Présence de métastases } \\
\text { viscérales extra-pulmonaires } \\
\text { ou } \\
\begin{aligned} \text { AFP }>10000 \mathrm{ng} / \mathrm{ml} \\
\text { ou } \\
\text { HCG }>50000 \mathrm{UI} / \mathrm{l} \\
\text { ou } \\
\text { LDH }>10 \times \mathrm{N}\end{aligned}\end{array}$ & $48 \%$ \\
\hline
\end{tabular}

AFP : Alphafoetoprotéine ; HCG : Hormone Chorionique Gonadotrope ; LDH : Lacticodéshydrogénase

\section{REFERENCES}

1. CULINE S., KRAMAR A., BIRON P., DROZ J.P. : Chemotherapy in adult germ cell tumors. Crit. Rev. Oncol. Hematol., 1996, 22 : 229-263.

2. DROZ J.P., CULINE S. : New prospects for the treatment of germ-cell tumours. Exp. Opin. Invest. Drugs, 1998, 7 : 11391157.

3. INTERNATIONAL GERM CELL CANCER COLLABORATIVE GROUP. International germ cell consensus classification : a prognostic factor-based staging system for metastatic germ cell cancers. J. Clin. Oncol., 1997, $15: 594-603$.

4. FLECHON A., CULINE S., DROZ J.P. : Intensive and timely chemotherapy, the key of success in testicular cancer ? Critic. Rev. Oncol. Hematol., 2001, 37 : 35-46.

\section{ABSTRACT}

\section{Chemotherapy in germ cell tumours of the testis Stéphane CULINE}

Germ cell tumours of the testis represent a model of curable disease, even in advanced stages. Cisplatin- based combination chemotherapy followed by surgical removal of residual metastatic disease has dramatically improved the prognosis of these patients, as about $90 \%$ of them are currently cured. The standard cytotoxic drugs used in first-line treatment are bleomycin, etoposide and cisplatin (the so-called BEP regimen). Treatment strategy is based on assessment of risk factors. Three cycles are generally used in patients with advanced pure seminoma or good-risk metastatic non-seminomatous tumours. Four cycles are required in patients with intermediate- or poor-risk metastatic non-seminomatous tumours, while two cycles of BEP constitute a safe and effective treatment option in early-stage tumours but with a high-risk of metastatic spread defined by a high percentage of embryonal carcinoma and/or the presence of vascular invasion. Cure rates as high as $90 \%$ are achieved provided risk factor strategies are accurately applied and standard treatment modalities are respected. All protocol violations can decrease the expected efficacy or induce unnecessary toxicity.

Key words: testicular cancer, germ cell cancer, chemotherapy 